\title{
Effect of Grain Morphology on Gas Bubble Swelling in UMo Fuels - a 3D Microstructure Dependent Booth Model
}

\author{
Shenyang Hu, Douglas Burkes, Curt. A Lavender, and Vineet Joshi \\ Pacific Northwest National Laboratory, P. O. Box 999, Richland, WA 99352
}

\begin{abstract}
A three dimensional microstructure dependent swelling model is developed for studying the fission gas swelling kinetics in irradiated nuclear fuels. The model is extended from the Booth model [1] in order to investigate the effect of heterogeneous microstructures on gas bubble swelling kinetics. As an application of the model, the effect of grain morphology, fission gas diffusivity, and spatially dependent fission rate on swelling kinetics are simulated in UMo fuels. It is found that the decrease of grain size, the increase of grain aspect ratio for the grain having the same volume, and the increase of fission gas diffusivity (fission rate) cause the increase of swelling kinetics. Other heterogeneities such as second phases and spatially dependent thermodynamic properties including diffusivity of fission gas, sink and source strength of defects could be naturally integrated into the model to enhance the model capability.
\end{abstract}

Keywords: UMo metal fuels; gas bubble swelling; grain morphology; fission rate; the Booth model 


\section{Introduction}

The development of low-enriched uranium (LEU) fuels such as $\gamma$ UMo dispersion and monolithic fuels is part of a global effort on nuclear non-proliferation [2]. Although $\gamma$ UMo fuels have the advantage of high density, excellent irradiation performance and good thermal conductivity, the volumetric swelling in irradiated metallic fuels is known to be an important design parameter because it affects not only the thermal conductivity of the fuels but also the mechanical integrity of fuel structures [3-5]. Nuclear reactions continuously generate fission gas atoms such as $\mathrm{Xe}$ and $\mathrm{Kr}$. These gas atoms segregate and form gas bubbles because of their extremely low solubility in UMo fuels. The nucleation and growth of fission gas bubbles are primarily responsible for the swelling in metallic fuels. A series of materials processes such as casting, mechanical rolling and thermal treatment are used in fuel fabrication [6, 7]. The resulting microstructure, such as grain size distribution, grain aspect ratio, Mo spatial distribution, porosity, dislocation density and structure, and second phase volume fractions, strongly depends on the material processes and process parameters. Therefore, a fundamental understanding of how fabricated microstructures and irradiation conditions affect the nucleation of gas bubbles and gas bubble swelling kinetics is essential for optimizing material processes to obtain desired microstructure and fuel performance.

Great efforts have been made in the development of modeling capability for predicting gas bubble swelling kinetics [1, 2, 5, 8-17] and fission gas release kinetics [18-21] in nuclear fuels. Booth [1] treated an individual grain within the material as a sphere whose boundary behaves as a perfect sink, and first developed a mathematical description of the diffusion of gas atoms in the representative sphere. Speight [8] extended the Booth model to consider the influence of trapping and re-dissolving of gas at intra- and inter-granular bubbles on the migration of fission gas atoms. General expressions are derived for the gas atom flux to grain boundaries and the gas atom concentration existing instantaneously within bubbles and in solution. Hayns and 
Wood et al. [9-11, 16] introduced the feature of intra- and intergranular gas bubble nucleation into the Booth model. Rest et al. [12-15] derived an analytical solution of intra- and inter- granular gas bubble size evolution by coupling rate equations that describe the nucleation and growth of inter- and intragranular bubbles under the simultaneous effect of irradiation-induced gas-atom re-solution. Recently, the effect of recrystallization on gas bubble swelling was also taken into account in UMo metallic fuels $[5,14]$. However, these models are based on the assumptions that the inter- and intra-granular gas bubble sizes are independent of position and only depend on time or fission density. In other words, the size and/or size distribution evolution of intra- and inter-granular gas bubbles only depends on the average grain size of the polycrystalline materials and irradiation conditions. Therefore, these models are applicable to a material with uniform microstructure such as equiaxed grains with homogeneous defect distributions, homogeneous thermodynamic and kinetic properties of defects, and uniform radiation conditions.

This work extended the capability of the Booth model by taking into account the effect of heterogeneous microstructures and inhomogeneous thermodynamic properties of defects on fission gas diffusion field, hence gas bubble swelling kinetics, in order to assess the effect of more realistic microstructures and accurate material properties on fuel performance. Our model employs all the evolution equations in the Booth model including fission gas concentration, the radius, density, and the content of fission gas atoms in inter- and intra-granular gas bubbles. However, our model releases the assumption of spatial independence of variables. Like the Booth model, our model assumes that the grain boundary is the perfect sink; fission gas has high mobility on grain boundaries; and fission gas concentration on grain boundaries remains in thermodynamic equilibrium. Experiments and modeling show that recrystallization in irradiated UMo fuels importantly affect the swelling kinetics [5, 15]. However, the recrystallization mechanisms and kinetics are not well understood. Therefore, in this work, we focus on studying the effect of the heterogeneous microstructures such as rolling-induced elongated grains, inhomogeneous grain size 
distribution (coarse grains and recrystallized grains), and casting-induced Mo homogeneity on the gas bubble swelling kinetics prior to recrystallization. The development of a swelling model integrating the effect of dynamic recrystallization on swelling is underway.

\section{Diffusion model of fission gas atoms inside a polycrystalline material}

We consider an irradiated polycrystalline UMo fuel. The nuclear reaction continuously generates fission gas atoms such as $\mathrm{Kr}$ and $\mathrm{Xe}$. Due to the extremely low solubility of fission gas atoms in the matrix phase, they precipitate to intra-granular gas bubbles and inter-granular gas bubbles. In the Booth model and extended models $[8,14,22]$, the polycrystalline material is treated as an equivalent sphere that has average grain size and whose boundary behaves as a perfect sink of fission gas atoms. The diffusion of fission gas atoms inside a spherical grain with distributed small intra-granular gas bubbles is described as

$$
\frac{\partial c(r, t)}{\partial t}=\nabla D_{g} \nabla c(r, t)+Y \dot{f}(t)-16 \pi f_{n} D_{g} r_{g} c_{g}(t)^{2}-4 \pi r_{b}(t) D_{g} c_{g}(t) c_{b}(t)+b n_{b}(t) c_{b}(t)
$$

where

$c(r, t)$ is the concentration of total gas atoms $(\mathrm{Xe}, \mathrm{Kr}, \ldots)$ in the UMo matrix.

$r$ is radial distance in spherical coordinates.

$t$ is the time.

$c_{b}(t)$ is the intra-granular bubble density.

$n_{b}(t)$ is the gas content per bubble.

$r_{b}(t)$ is the radius of the intra-granular gas bubble.

$c_{g}(t)$ is the volumetric average concentration of the dissolved gas. 
$\dot{f}$ is the fission rate.

$Y$ is the gas atom yield per fission.

$b$ is the radiation-induced resolution rate of intra-granular gas bubble.

$f_{n}$ is the nucleation factor.

$D_{g}$ is the diffusion coefficient gas atom under irradiation.

$r_{g}$ is the radius of the gas atom.

The first term on the right hand side of equation (1) describes gas atom diffusion driven by the concentration gradient; the second term is the source term describing the generation of gas atoms during a fission reaction; the third and fourth terms present the loss of gas atoms associated with gas bubble nucleation and absorption at gas bubbles, respectively; and the last term is the gain of gas atoms associated with the radiation-induced resolution of gas bubbles. For simplicity, the local gas concentration $c(r, t)$ in the third and fourth terms is replaced by the volumetric average concentration of the dissolved gas $c_{g}(t)$. Local gas bubble structural variables including the density, radius and gas content in the bubbles are assumed to be the spatial average inside the representative sphere. Therefore, the source and sink terms are not spatially dependent.

In a polycrystalline material the intra-granular gas bubble structure might strongly dependent upon microstructure. For example, the gas bubble structure at the center of a large grain could be different from that near the grain boundary. In UMo fuels, the gas bubbles inside large grains often form a $f c c$ superlattice while the gas bubble superlattice structure is unstable inside small grains [2, 23]. Therefore, the grain size, grain aspect ratio, and structural defects might result in a heterogeneous gas bubble structure, hence the gas bubble swelling kinetics. In order to study the effect of the heterogeneous microstructure on gas diffusion, gas bubble evolution, and gas bubble swelling, we treat all the variables in equation (1) as a function of time and space in 
this work. The driving force of fission gas diffusion is generalized to be the local chemical potential gradient. Thus the effect of local stress fields, and structural defects such as grain boundaries on diffusion driving force could be naturally taken into account $[24,25]$. The fission rate, intra-granular gas bubble density, radius and the gas content in gas bubbles are time and space dependent functions. The diffusion equation (1) is generalized as

$$
\begin{aligned}
\frac{\partial c(\mathbf{r}, t)}{\partial t} & =\nabla D_{g} \nabla \mu(\mathbf{r}, t)+Y \dot{f}(\mathbf{r}, t)-16 \pi f_{n} D_{g} r_{g} c(\mathbf{r}, t)^{2} \\
& -4 \pi r_{b}(\mathbf{r}, t) D_{g} c(\mathbf{r}, t) c_{b}(\mathbf{r}, t)+b n_{b}(\mathbf{r}, t) c_{b}(\mathbf{r}, t)
\end{aligned}
$$

where $\mathbf{r}$ is the coordinate and $\mu$ is the chemical potential of fission gas atoms. Grain boundaries are assumed to be a perfect sink similar to the Booth model. It is assumed that the concentration of fission gas atoms remain in thermal equilibrium concentration $c_{G B}^{e q}$ considering the fact that the diffusivity of gas atoms on grain boundaries are much larger than those inside grains, and the large inter-granular gas bubbles are strong sinks for the gas atoms. For a given intra-granular gas bubble microstructure $\left(n_{b}(\mathbf{r}, t), c_{b}(\mathbf{r}, t), r_{b}(\mathbf{r}, t)\right)$ and the equilibrium concentration $\left(c_{G B}^{e q .}\right)$ of gas atoms on the grain boundaries, solving equation (2) we obtain the total flux of gas atoms to the grain boundaries and the evolution of gas atom concentration $c(\mathbf{r}, t)$ inside the grains.

\section{Evolution of intra- and inter-granular gas bubbles}

A similar model presented by Spino et. al. for $\mathrm{UO}_{2}$ fuels [22] is employed to describe inter- and intra- granular gas bubble evolution in UMo fuels for known local gas concentration and the total flux of gas atoms to grain boundaries. At the operation temperature of metallic UMo fuels $(\mathrm{T}<500 \mathrm{~K})$, the diffusion of fission gas atoms is assumed to be athermal with the gas atom diffusivity $D_{g}$ proportional to the fission rate $\dot{f}$. The gas atom resolution rate $b$ is also assumed proportional to the fission rate $\dot{f}$ [13]. The intra-granular gas bubbles are assumed as identical inside a 
representative volume $(\mathrm{dx} * \mathrm{dy} * \mathrm{dz})$ and the rate equation describing the time evolution of the density of gas atoms in intra-granular bubbles can be described by

$$
\frac{\partial n_{b}(r, t) c_{b}(\mathbf{r}, t)}{\partial t}=16 \pi f_{n} D_{g} r_{g} c(\mathbf{r}, t)^{2}+4 \pi r_{b}(\mathbf{r}, t) D_{g} c(\mathbf{r}, t) c_{b}(\mathbf{r}, t)-b n_{b}(\mathbf{r}, t) c_{b}(\mathbf{r}, t)
$$

The three terms on the right hand side of equation (3) present, respectively, the change in the density of gas atoms in intra-granular gas bubbles due to bubble nucleation, the gas bubble growth by absorbing gas atoms in the matrix, and the loss of gas atoms from bubbles due to irradiation-induced re-solution. Equation (3) can be separated into two equations describing the time evolution of the fission gas bubble density and of the gas content per gas bubble, as follows:

$$
\begin{gathered}
\frac{\partial c_{b}(\mathbf{r}, t)}{\partial t}=\frac{16 \pi f_{n} D_{g} r_{g} c(\mathbf{r}, t)^{2}}{n_{b}(\mathbf{r}, t)}-\frac{b c_{b}(\mathbf{r}, t)}{2} \\
\frac{\partial n_{b}(\mathbf{r}, t)}{\partial t}=4 \pi r_{b}(\mathbf{r}, t) D_{g} c(\mathbf{r}, t)-\frac{b n_{b}(\mathbf{r}, t)}{2}
\end{gathered}
$$

Under the assumption of thermal equilibrium, the radius of intra-granular gas bubbles $r_{b}(\mathbf{r}, t)$ is related to $n_{b}(\mathbf{r}, t)$ through the gas law and the capillarity relation. Using a modified Van der Waals gas law,

$$
\frac{2 \gamma}{r_{b}(\mathbf{r}, t)}\left(\frac{4}{3} \pi r_{b}^{3}(\mathbf{r}, t)-h_{s} b_{v} n_{b}(\mathbf{r}, t)\right)=n_{b}(\mathbf{r}, t) k T
$$

where $\gamma$ is the surface tension, $b_{v}$ is the Van der Waals constant for $\mathrm{Xe}, k$ is Boltzmann's constant, $T$ is the absolute temperature, and $h_{s}$ is a fitting parameter that for a given $T$ makes equation (6) equivalent to the hard-sphere equation of state [26]. For nanometer sized gas bubbles, we have an approximate solution to equation (6) as

$$
r_{b}(\mathbf{r}, t)=\left(\frac{3 h_{s} b v n_{b}(\mathbf{r}, t)}{4 \pi}\right)^{1 / 3}
$$

Therefore, the radius of intra-granular gas bubbles can be calculated by equation (7) 
once we know the solution of equation (5).

The grain boundary acts as a perfect sink for fission gas atoms. Since there are plenty of gas bubble heterogeneous nucleation sites on grain boundaries, and the mobility of gas atoms on grain boundaries is usually large, it is assumed that the density, radius and gas content of inter-granular gas bubbles is independent of location. With this assumption, inter-granular gas bubble density in irradiated materials can be calculated as $[10,15,22]$

$$
C_{b}(t)=\left(\frac{8 z a K}{12^{1 / 3} \pi^{2} \xi D_{g} \delta}\right)^{1 / 2}
$$

where

$a$ is the lattice constant.

$z$ is the number of sites explored per gas-atom jump.

$\xi$ is the grain-boundary diffusion enhancement factor.

$\delta$ is the width of the boundary.

$K$ is the flux of gas atoms from grain interior to the grain boundary.

In equation (8) $\xi$ is a major fitting parameter. For UMo fuels, the results $[10,15,22]$ show that a value from 15 to 125 can reasonably reproduce existing experimental data [13]. Heterogeneous microstructures may cause a non uniform gas flux, hence a heterogeneous inter-granular gas bubble structure. Since the developed model directly solves the diffusion equation in polycrystalline materials it enables one to calculate the spatially dependent fission gas flux $K(\mathbf{r}, t)\left[a t o m s / m^{2} s\right]$ from grain interior to the grain boundary. Using $K(\mathbf{r}, t)\left[\right.$ atoms $\left./ m^{2} s\right]$ we can study the heterogeneous inter-granular gas bubble structure evolution if necessary. The nucleation kinetics may also affect the gas bubble structures such as the gas bubble size distribution. Rest et al. [14] developed a model to predict the evolution of fission gas bubble size distribution on grain boundary in UMo fuels. It is also possible to extend the current 
model to study the gas bubble size distribution evolution by integrating the method [14].

Solving equations (2), (4-5) and (7) together with the equilibrium concentration of gas atoms at grain boundaries, we have the average gas flux $K(t)\left[\right.$ atoms $\left./ m^{2} s\right]$. The total amount $N(t)\left[\right.$ atoms $\left./ \mathrm{m}^{2}\right]$ of gas atoms on the grain boundary can be calculated by integrating the gas flux as $N(t)=\int_{0}^{t} K(t) d t$. The content of gas atoms per inter-granular gas bubble can be calculated by $N_{b}(t)=N(t) / C_{b}(t)$.

The modified Van der Waals gas law in equation (6) can also be used to estimate the radius of the intergranular gas bubbles. For a finite size of gas bubble, the radius of an inter-granular gas bubble is obtained from the solution to equation (6), i.e.,

$$
\begin{aligned}
R_{b}(t) & =\left[\frac{3 h_{s} b_{v} N_{b}(t)}{8 \pi}+\sqrt{\left(\frac{3 h_{s} b_{v} N_{b}(t)}{8 \pi}\right)^{2}-\left(\frac{N_{b}(t) k T}{8 \pi \gamma}\right)^{3}}\right]^{1 / 3} \\
& +\left[\frac{3 h_{s} b_{v} N_{b}(t)}{8 \pi}-\sqrt{\left(\frac{3 h_{s} b_{v} N_{b}(t)}{8 \pi}\right)^{2}-\left(\frac{N_{b}(t) k T}{8 \pi \gamma}\right)^{3}}\right]^{1 / 3}
\end{aligned}
$$

\section{Gas bubble swelling before the grain recrystallization}

The intra- and inter- granular gas bubble evolution equations presented above can be used to assess the fission gas swelling in irradiated fuels. The fractional swelling due to fission gas is thus given by

$$
\frac{\Delta V(t)}{V}=\left[\int_{V} \frac{4 \pi}{3} r_{b}^{3}(\mathbf{r}, t) c_{b}(\mathbf{r}, t) d V+\frac{4 \pi}{3} R_{b}^{3}(t) C_{b}(t) S+\int_{V} \varepsilon_{0} c_{g}(\mathbf{r}, t) d V\right] / V
$$

where $V$ is the volume of the simulation cell. The first term at the right hand side of equation (10) accounts for the contribution of distributed intra-granular gas bubbles, the second term for the contribution of inter-granular gas bubbles, and the third term 
for the contribution of distributed gas atoms in the matrix. $S$ is the total area of grain boundaries in the polycrystalline materials and $\varepsilon_{0}$ is the formation volume of gas atom. Both experiments and theory $[5,14,15]$ demonstrated that recrystallization in UMo fuels plays an important role in fuel swelling. Experiments show that recrystallization takes place when the fission density is larger than a critical value $\left(3.1 \sim 3.5 \times 10^{27}\right.$ fission $\left./ \mathrm{m}^{3}\right)$ [14]. In this work we focus on investigating the effect of initial grain morphology on swelling kinetics prior to recrystallization.

\section{Results and discussions}

The simulations are carried out in a three dimensional (3D) polycrystalline domain $200 d x \times 200 d y \times 200 d z$, where $d x, d y, d z$ are the grid sizes in $\mathrm{x}-, \mathrm{y}-$ and $\mathrm{z}-$ axis, respectively. Periodic boundary conditions are applied in $\mathrm{x}-, \mathrm{y}-$ and $\mathrm{z}-$ directions. Images of 3D polycrystalline structures can be directly used as the initial structure. Unfortunately, there is no 3D image of polycrystalline UMo available. In addition, it is very challenging to reconstruct 3D polycrystalline structures using $2 \mathrm{D}$ SEM and/or EBSD images. Therefore, in this work, a phase-field model of grain growth is employed to create a $3 \mathrm{D}$ polycrystalline structure as the initial structure for the simulations. For a given polycrystalline structure, the grain boundary is first identified where a chemical potential $\mu_{G B}$ of fission gas atoms could be applied that is an additional driving force for fission gas diffusion to grain boundaries besides the fission gas concentration gradient. The total area $S$ of grain boundaries, which is used in equations (10), is calculated; and an equilibrium concentration of fission gas on the grain boundaries is updated every 10 time steps in the simulations. Table 1 lists the model parameters that are used in literature $[2,13-15,22,27]$. Different sets of Xe diffusivity, fission rates, and nucleation factors are used to study their effect on the swelling kinetics.

For given model parameters, solving the equations (3), we can have the time 
evolution of intra-granular gas bubbles $(4,5,7)$; using equation (8), we can calculate the inter-granular gas bubble evolution, and then the swelling can be calculated by equation (10).

Table 1. Model parameters used in the simulations

(Insert Table 1 here)

\subsection{Effect of grain morphology on gas bubble microstructure}

The Booth model used average intra-granular gas bubble morphology (average gas bubble concentration, radius, and gas content in bubbles) as model variables for assessing the swelling kinetics. First we present the intra-granular gas bubble evolution in a polycrystalline structure with 548 grains and an average grain size $1.34 \mu m$ in diameter to demonstrate the capability of our model. Xe diffusivity, fission rates, nucleation factors and the equilibrium fission gas concentration $c_{G B}^{e q}$ used in the simulations are $D_{0}=1.0 \times 10^{-40} \mathrm{~m}^{5} /$ fission,$\dot{f}=1.2 \times 10^{20}$ fission $/\left(\mathrm{m}^{3} \mathrm{~s}\right)$, $f_{n}=0.03$ and $b=2.4 \times 10^{-4}$, respectively. Figure 1 shows the distribution of intra-granular gas bubble density $\left(\right.$ bubbles $\left./ \mathrm{m}^{3}\right)$, gas content (the number of Xe atoms per gas bubble), and gas bubble radius ( $\mathrm{nm}$ ) inside gas bubbles on the plane A at time $t=672$ hours. The color bar shows the value of the variables. For instance, red regions in Figure 1b denote high gas bubble density while blue regions denote low gas bubble density. It can clearly be seen that the gas bubble density, radius, and gas content in bubbles decrease from the center of grains to grain boundaries. The spatial distributions of gas bubble density, radius, and gas content inside grains were analyzed. The results show that 1) the maximum gas bubble density, radius, and gas content inside grains increases with increased grain size, and 
2) average gas bubble density, radius, and gas content inside grains also increase as the grain size increases. Figure $2 \mathrm{a}$ shows the grain size dependence of average gas bubble density inside grains at time $t=1157$ and 2315 hours, respectively. The time evolution of gas bubble density on the line B-B' shown in Figure 1b is plotted in Figure $2 \mathrm{~b}$. The results show that the increment of gas bubble density inside grains monotonically decreases, and the gas bubble structure gradually reaches a steady state structure. The temporal evolution and grain size dependence of radius and gas content inside grains have the same tendencies as that shown in Figure 2. At steady state the intra-granular gas bubble density and radius inside grains increases with increased grain size. The predicted gas bubble density and diameter inside large grains is about $1.8 \times 10^{24}$ bubble $/ \mathrm{m}^{3}$ and $2.2 \mathrm{~nm}$, respectively. TEM results of intra-granular gas bubble structures in UMo fuels shows that a nano sized gas bubble super-lattice forms inside grains [23, 28, 29]. The gas bubble density is around $1.0 \sim 2.6 \times 10^{24}$ bubble $/ \mathrm{m}^{3}$, and the gas bubble size is about $2 \sim 3.5 \mathrm{~nm}$ in diameter.

No large change in the gas bubble density and only a slight increase of gas bubble size were observed with increasing fission density. From the comparison it can be seen that the intra-granular gas bubble structure obtained from simulations are in acceptable agreement with the experimental results. In addition, our model not only predicts overall gas bubble evolution kinetics including the average gas bubble density and size, but can also provide more thorough insight of heterogeneous gas bubble structures in irradiated materials than the Booth model.

(Insert Figure 1 here)

Figure 1. (a) a simulated polycrystalline UMo structure with 548 grains and average grain size $1.34 \mu m$ in diameter, (b) the distribution of intra-granular gas bubble density, (c) the distribution of gas content in gas bubbles, (d) the distribution of gas 
bubble radius on the plane A shown in Figure 1(a).

(Insert Figure 2 here)

Figure 2. (a) Grain size dependence of average intra-granular gas bubble density for two different times. (b) Temporal evolution of intra-granular gas bubble density along the BB' line shown in Figure $1 \mathrm{~b}$.

\subsection{Comparison of gas bubble swelling kinetics}

Our model is an extension of the Booth model. It is expected that two models should predict similar swelling kinetics if the polycrystalline structure has equiaxed grains because such a polycrystalline structure could be reasonably described by a representative spherical grain in the Booth model. To verify this, we consider two polycrystalline structures with equiaxed grains. The average grain sizes are $1.34 \mu \mathrm{m}$ and $4.63 \mu \mathrm{m}$ in diameter, respectively. The parameters: Xe diffusivity, fission rates, and nucleation factors are $D_{0}=1.0 \times 10^{-40} \mathrm{~m}^{5} /$ fission, $\dot{f}=1.2 \times 10^{20}$ fission $/\left(\mathrm{m}^{3} \mathrm{~s}\right)$ and $f_{n}=0.01$, respectively. The equilibrium fission gas concentration $c_{G B}^{e q}$ is an additional parameter in our model compared to the Booth model. Figure 3 presents the swelling kinetics obtained from the Booth model and our microstructural dependent swelling model for different equilibrium fission gas concentration $c_{G B}^{e q}$ on grain boundaries. Increasing the fission gas equilibrium concentration $c_{G B}^{e q}$ means decreasing the concentration gradient, hence, decreasing the diffusion driving force and gas flux from the grain interior to grain boundaries. As expected, the results in Figure 3 show that the swelling kinetics decreases with the increase of the equilibrium fission gas concentration $c_{G B}^{e q}$. For small and large grain polycrystalline structures, the swelling kinetics predicted by our model approaches that from the Booth model when the equilibrium fission gas concentration on the grain boundaries increases from 
$5.0 \times 10^{-6}$ to $1.5 \times 10^{-5}$. However, the simulations confirm that increasing or decreasing the Xe solubility will not further affect the swelling kinetics. In other words, the symbol lines for $c_{G B}^{e q}=5.0 \times 10^{-6}$ and $c_{G B}^{e q}=1.5 \times 10^{-5}$ give the upper and lower bounds of swelling kinetics, respectively. Since the Booth model uses a representative spherical grain while our model uses a three dimensional polycrystalline structure, the difference in grain shape and grain size distribution used in two models causes different Xe fluxes to grain boundaries, hence, the difference in swelling kinetics. But comparing the results in Figure 3 we can conclude that 1) the swelling kinetics predicted from our model are in good agreement with those from the Booth model, and 2) the Booth model underestimates the swelling kinetics due to the simplified assumption of grain morphology. The Xe solubility that is a parameter in our model can be estimated by Xe formation energies at grain boundaries in UMo alloys. Unfortunately, there is no such data available in literature to the authors knowledge. Henceforward, the equilibrium fission gas concentration on the grain boundaries will be set to be $1.5 \times 10^{-5}$ because it produces the best agreement with the Booth model and the swelling kinetics are not sensitive to the Xe solubility.

(Insert Figure 3 here)

Figure 3. A comparison of swelling kinetics obtained from the Booth model and our model in two polycrystalline structures.

For intra-granular gas bubbles, the spatial average of gas bubble density and radius are calculated. The results from our model and the Booth model are plotted in Figure 4. Our model predicted a higher inter-granular gas bubble density and lower intra-granular gas bubble density than that predicted by the Booth model, hence, a larger swelling kinetics. These results are consistent with the analysis above. However, 
if comparing the time evolution of gas bubble density and radius, we can find that the two models give very similar evolution kinetics. For example, the inter-granular gas bubble density attains the steady state quickly (Figure 4b) while the intra-granular gas bubble density attains the steady state slowly (Figure 4a). For gas bubble radius, our model predicts a smaller radius of inter-granular gas bubble density (Figure 4c) and larger radius of intra-granular gas bubbles than that from the Booth model (Figure 4d).

Although the two models produce similar swelling kinetics, the difference in gas bubble densities and size is obvious. For example, about $30 \%$ difference is observed for an average intra-granular gas bubble radius. The Booth model solves the fission gas diffusion and gas bubble evolution in a representative sphere. Our model solves the fission gas diffusion field in a polycrystalline structure. Due to the presence of small grains, the fission gas flux calculated in our model is larger than that from the Booth model, which results in a larger nucleation rate of inter-granular gas bubbles, and all the gas bubble structure difference. Therefore, the Booth model will underestimate the swelling kinetics, especially in a polycrystalline structure with a broad grain size distribution.

(Insert Figure 4 here)

Figure 4. Comparison of average gas bubble density and size evolution obtained from the Booth model and our model in two polycrystalline structures. (a) Intra-granular gas bubble density, (b) Inter-granular gas bubble density, (c) Radius of intra-granular gas bubbles, and (d) Radius of inter-granular gas bubbles.

5.3 Effect of grain morphology on gas bubble swelling kinetics

Four crystal structures are generated by the phase-field model of grain growth for 
studying the effect of grain morphology on swelling kinetics. Crystal A has small grains. Crystal B has large grains. Crystals C and D have elongated grains with aspect ratios 4:1 and 16:1, respectively. These elongated grains mimic rolling-induced grain morphological changes and have the same volume as that in Crystal B. The grain morphology of these crystal structures are listed in Table 2.

Table 2 Morphology of four polycrystalline structures (Insert Table 2 here)

The predicted effect of grain morphology on swelling kinetics is presented in Figure 5. Two obvious tendencies are observed: 1) the swelling kinetics increase with decreasing grain size, and 2) the swelling kinetics increase with increasing aspect ratio for grains with the same volume. With the grain morphology changes from large grain to small grain and/or from equiaxed grain to elongated grain, the diffusion path of fission gas becomes shorter and shorter, which causes an increase of fission gas flux to grain boundaries and a decrease of fission gas concentration inside grains. As a consequence, the intra-granular gas bubble density and radius decreases, the intra-granular gas bubble structure more quickly reaches steady state, and swelling kinetics increases due to the increasing contribution of inter-granular gas bubbles to swelling. These results demonstrate that the developed model extends the Booth model's capability, and enables one to study the effect of grain morphology on swelling. The model can be further extended to consider the effect of any heterogeneous structures such as the distributed second phase that may have different swelling mechanisms, e.g., the $\alpha$ and $\alpha^{\prime}$ phases, dislocations, recrystallization zone, and heterogeneous fission rate on swelling kinetics.

(Insert Figure 5 here) 
Figure 5. The effect of grain morphology on gas bubble swelling kinetics under fission rate $\dot{f}=2.4 \times 10^{22}$ fission $/\left(m^{3} s\right)$ and nucleation factor $f_{n}=0.02$.

5.4 Effect of fission rate and Xe diffusivity on gas bubble swelling kinetics Xe diffusivity in irradiated materials is assumed to be proportional to fission rate written as $D_{X e}=D_{0} \dot{f}[30] . \quad D_{0}$ is a constant of proportionality associated with the volume affected by a fission spike. For UMo fuels it is taken to be $D_{0}=1.0 \times 10^{-40} \mathrm{~m}^{5}$ [15]. Therefore, Xe diffusivity is directly related to the fission rate. Gas bubble swelling is simulated in the four crystal structures listed in Table 2 for different $\mathrm{Xe}$ diffusivity or fission rates. Figure 6a presents the result in Crystal B that has large grains. It is found that the swelling kinetics increase with increasing Xe diffusivity/fission rate. The swelling in terms of fission density, that is fission rate multiplied by time, is replotted in Figure 6b. It clearly shows that the total amount of swelling is determined by the fission density for a given crystal structure. The effect of fission rates on swelling kinetics for Crystals B, C and D were also studied. The results reveal the similar grain morphological dependence shown in Figure 5 for different fission rates, i.e., 1) the swelling kinetics increase with decreasing grain size, and 2) the swelling kinetics increase with increasing aspect ratio for grains with the same volume.

(Insert Figure 6 here)

Figure 6 Effect of fission rates on gas bubble swelling kinetics in Crystal B. (a) swelling vs time, and (b) swelling vs fission density. 
5.5 Effect of Mo inhomogeneous distribution on gas bubble swelling kinetics

A core shell structure of Mo distribution, which has higher Mo concentration at the center of grains and lower Mo concentration at the grain boundaries, often forms during casting of metallic UMo fuels due to a strong temperature dependence of Mo solubility in $\gamma$ UMo. From experiments the Mo concentration varies anywhere between 10 at $\%$ to 25 at $\%[31,32]$. This Mo inhomogeneity might cause inhomogeneous distribution of $U^{235}$, which may result in spatially dependent fission rate. In addition, the material process might induce other inhomogeneities such as second phase UC particle distribution, formation of alpha U phase, and dislocation networks associated with cold and hot rolling. These heterogeneous structures will affect fission gas diffusivity and nucleation rate, hence the gas bubble swelling kinetics. If we introduce the inhomogeneous model parameters related to the structure and thermodynamic property inhomogeneity, the current model is able to study the influence of heterogeneous structures and inhomogeneous properties on swelling kinetics. As an example of the application, we consider the effect of spatially dependent fission rate on swelling related to $U^{235}$ inhomogeneous distribution. A phase-field model of solute diffusion in a polycrystalline structure is used to create a core shell structure of Mo distribution. The Mo distribution is converted to $U^{235}$ distributions based on the lattice conservation and $U$ enrichment. Then we assumed that the fission rate is proportional to the $U^{235}$ concentration. Figure 7 a shows the core shell structure of Mo distribution obtained from the phase-field model in Crystal B. The color bar presents Mo atoms per unit volume $\left(\mathrm{m}^{3}\right)$ in U10wt\% Mo alloys. Three different Mo distributions, which are denoted as Case1, Case 2, and Case 3, are created for studying the effect of Mo inhomogeneity/fission rate on swelling. Mo has a uniform distribution in the polycrystalline structure in Case 1. The inhomogeneity of Mo distribution in Case 3 is stronger than that in Case 2. The fission rate is written as $\dot{f}(\mathbf{r})=F(\mathbf{r}) \dot{f} . \quad F(\mathbf{r})$ is the scale factor of fission rate that is a function of local Mo 
concentration. In order to have the same average fission rate and fission density in all Cases $F(\mathbf{r})$ satisfies the equation $\int_{V} F(\mathbf{r}) d V / V=1$, where $V$ is the volume of simulation cell. Figure $7 \mathrm{~b}$ presents the scale factors of fission rate along the BB' line as shown in Figure 7a for Case1, Case 2 and Case3. $F(\mathbf{r})$ is equal to 1 in Case 1 that means a homogeneous fission rate. The fission rate near grain boundaries is larger than at the center of grains.

(Insert Figure 7 here)

Figure 7. (a) Mo spatial distribution on plane A which is generated by phase-field modeling in Crystal B of U10wt\% Mo fuels, (b) scale factor of fission rate along the BB' line shown in (a) for three different Mo distributions.

The simulation results are shown in Figure 8. It can be seen that swelling kinetics increase with increasing fission rate inhomogeneity from Case 1, Case 2 to Case 3 for crystal structures with small and large grains, respectively. However, the influence of fission rate inhomogeneity on swelling is not significant. In Case 3 the fission rate at the center of the grain is about $0.71 \dot{f}$ while the fission rate at the grain boundaries is about $1.22 \dot{f}$. Such an inhomogeneous fission rate causes about $6.5 \%$ swelling increase under fission rate $\dot{f}=1.2 \times 10^{20}$ fission $/\left(\mathrm{m}^{3} \mathrm{~s}\right)$ after 900 hours of irradiation. Besides the impact of Mo inhomogeneity on the fission rate, Mo inhomogeneity may also cause the phase transition from gamma $U$ to alpha $U$ and affect the recrystallization process, which may impact the fuel swelling and fuel performance.

(Insert Figure 8 here) 
Figure 8. Effect of Mo inhomogeneous distributions on swelling kinetics under fission rate $\dot{f}=1.2 \times 10^{20}$ fission $/\left(m^{3} s\right)$ and nucleation factor $f_{n}=0.03$.

\section{Conclusions and remarks}

A microstructure dependent gas bubble swelling model is developed that is extended from the Booth model. The model enables one to investigate the effect of heterogeneous microstructures such as grain size distribution, and grain aspect ratio, and spatially dependent thermodynamic properties including diffusivity of fission gas, sink and source strength of defects, temperature, stresses and chemical potential on gas bubble swelling kinetics. As application of the model, the effect of grain morphology, fission gas diffusivity, spatially dependent fission rates on swelling kinetics were simulated. It was found that 1) swelling kinetics increase with decreasing grain sizes. 2) swelling kinetics increase with increasing grain aspect ratio for grains having the same volume. 3) swelling kinetics increase with increasing fission gas diffusivity (fission rate), and 4) the total amount of swelling is determined by the fission density under the assumptions that the fission gas diffusivity, gas bubble nucleation factor, and dissolution strength are proportional to the fission rate. It was found that the effect of the spatially dependent fission rate related to inhomogeneous Mo distribution on swelling kinetics is not significant under simulation conditions such as grain size around a few micrometers and before recrystallization.

The simulations demonstrate that the developed model dramatically extends the prediction capability of the Booth model. However, for engineering applications, the model needs to be further improved. In current simulations, the grain sizes are around a few micrometers that are much smaller than those (approximately few tens of micrometers) in monolithic UMo fuels. The limitation is from the assumption that the thickness of gas bubble denuded zone on the grain boundary is set to be the characteristic length $l_{0}$. All the fission gas atoms diffusing into the gas bubble 
denuded zone will be absorbed by the inter-granular gas bubble. The grid size $d x=d y=d z$ in the simulation cell is set to be $l_{0}$. Thus the simulation size is limited by the characteristic length $l_{0}$ and current computer capability. For a given $l_{0}$, we may use an adaptive mesh, smaller mesh on grain boundaries and larger mesh inside the grain, to largely increase the simulation size. The recrystallization is critical to UMo fuel swelling and performance. The current model is applicable before recrystallization occurs. Experiments show that the recrystallization is related to the local radiation defect accumulation such as dislocation loop density [33-36].

Therefore, the heterogeneous microstructures such as grain morphology will affect the the evolution of dislocation loop density, hence, recrystallization and the swelling kinetics. The work integrating the recrystallization kinetics into the swelling model is underway. In addition, the developed model is able to study the effect of sink and sources of defects on the swelling kinetics such as dislocations and grain boundaries. A predictive model needs the thermodynamic properties of defects including the sink strength and emission rates. 


\section{Acknowledgements}

The work described in this article was performed by Pacific Northwest National Laboratory, which is operated by Battelle for the United States Department of Energy under Contract DE-AC05-76RL01830. This study was supported by the U.S. Department of Energy, National Nuclear Security Administration, Office of Material Management and Minimization Reactor Conversion Program.

\section{References}

[1] A.H. Booth, in, Atomic Energy of Canada Limited Report No. 496, 1957.

[2] Y.S. Kim, G.L. Hofman, J Nucl Mater 419 (2011) 291-301.

[3] Y.S. Kim, G.L. Hofman, J.S. Cheon, A.B. Robinson, D.M. Wachs, J Nucl Mater 437 (2013) 37-46.

[4] R.M. Hengstler, L. Beck, H. Breitkreutz, C. Jarousse, R. Jungwirth, W. Petry, W. Schmid, J. Schneider, N. Wieschalla, J Nucl Mater 402 (2010) 74-80.

[5] Y.S. Kim, G.L. Hofman, J.S. Cheon, J Nucl Mater 436 (2013) 14-22.

[6] G. Moore, in: AFIP-2 Fabrication Summary Report, INL/EXT-08-14871, 2010.

[7] D.E. Burkes, A.M. Casella, E.C. Buck, A.J. Casella, M.K. Edwards, P.J. MacFarlan, K.N. Pool, B.D. Slonecker, F.N. Smith, F.H. Steen, R.E. Thornhill, in: Fuel Thermo-physical Characterization Project: Fiscal Year 2013 Final Report, PNNL-22981, 2013.

[8] M.V. Speight, Nucl Sci Eng 37 (1969) 180-\&.

[9] M.R. Hayns, M.H. Wood, P Roy Soc Lond a Mat 368 (1979) 331-343.

[10] M.H. Wood, K.L. Kear, J Nucl Mater 118 (1983) 320-324.

[11] M.H. Wood, J.R. Matthews, J Nucl Mater 91 (1980) 35-40.

[12] J. Rest, J Nucl Mater 402 (2010) 179-185.

[13] J. Rest, G.L. Hofman, Y.S. Kim, J Nucl Mater 385 (2009) 563-571.

[14] J. Rest, J Nucl Mater 407 (2010) 55-58.

[15] J. Rest, J Nucl Mater 346 (2005) 226-232.

[16] J.R. Matthews, M.H. Wood, J Nucl Mater 91 (1980) 241-256.

[17] Y. Cui, S.R. Ding, Z.T. Chen, Y.Z. Huo, J Nucl Mater 457 (2015) 157-164.

[18] R.J. White, M.O. Tucker, J Nucl Mater 118 (1983) 1-38.

[19] D.M. Dowling, R.J. White, M.O. Tucker, J Nucl Mater 110 (1982) 37-46.

[20] K. Forsberg, A.R. Massih, J Nucl Mater 127 (1985) 141-145.

[21] P.C. Millett, M.R. Tonks, S.B. Biner, J Nucl Mater 424 (2012) 176-182.

[22] J. Spino, J. Rest, W. Goll, C.T. Walker, J Nucl Mater 346 (2005) 131-144.

[23] J. Gan, D.D. Keiser, B.D. Miller, A.B. Robinson, J.F. Jue, P. Medvedev, D.M. Wachs, J Nucl Mater 424 (2012) 43-50.

[24] S.Y. Hu, C.H. Henager, H.L. Heinisch, M. Stan, M.I. Baskes, S.M. Valone, J Nucl Mater 392 (2009) 292-300.

[25] N. Moelans, B. Blanpain, P. Wollants, Phys Rev B 78 (2008).

[26] C. Ronchi, J Nucl Mater 96 (1981) 314-328.

[27] A. Denis, R. Piotrkowski, J Nucl Mater 229 (1996) 149-154.

[28] S. Van den Berghe, W. Van Renterghem, A. Leenaers, J Nucl Mater 375 (2008) 340-346.

[29] J. Gan, D.D. Keiser, D.M. Wachs, A.B. Robinson, B.D. Miller, T.R. Allen, J Nucl Mater 396 (2010) 234-239.

[30] H. Matzke, Radiat Eff Defect S 53 (1980) 219-242.

[31] Z.J. Xu, V. Joshi, S.Y. Hu, D. Paxton, C. Lavender, D. Burkes, J Nucl Mater 471 (2016) 154-164.

[32] K.H. Kim, D.B. Lee, C.K. Kim, G.E. Hofman, K.W. Paik, J Nucl Mater 245 (1997) 179-184.

[33] J. Rest, J Nucl Mater 349 (2006) 150-159.

[34] I.L.F. Ray, H. Thiele, H. Matzke, J Nucl Mater 188 (1992) 90-\&.

[35] K. Nogita, K. Une, Nucl Instrum Meth B 91 (1994) 301-306.

[36] J. Spino, D. Baron, M. Coquerelle, A.D. Stalios, J Nucl Mater 256 (1998) 189-196. 
Figure 1. (a) a simulated polycrystalline UMo structure with 548 grains and average grain size $1.34 \mu m$ in diameter, (b) the distribution of intra-granular gas bubble density, (c) the distribution of gas content in gas bubbles, (d) the distribution of gas bubble radius on the plane A shown in Figure 1(a).

Figure 2. (a) Grain size dependence of average intra-granular gas bubble density for two different times. (b) Temporal evolution of intra-granular gas bubble density along the BB' line shown in Figure $1 \mathrm{~b}$.

Figure 3. A comparison of swelling kinetics obtained from the Booth model and our model in two polycrystalline structures.

Figure 4. Comparison of average gas bubble density and size evolution obtained from the Booth model and our model in two polycrystalline structures. (a) Intra-granular gas bubble density, (b) Inter-granular gas bubble density, (c) Radius of intra-granular gas bubbles, and (d) Radius of inter-granular gas bubbles.

Figure 5. The effect of grain morphology on gas bubble swelling kinetics under fission rate $\dot{f}=2.4 \times 10^{22}$ fission $/\left(m^{3} s\right)$ and nucleation factor $f_{n}=0.02$. 
Figure 6 Effect of fission rates on gas bubble swelling kinetics in Crystal B. (a) swelling vs time, and (b) swelling vs fission density.

Figure 7. (a) Mo spatial distribution on plane A which is generated by phase-field modeling in Crystal B of U10wt\%Mo fuels, (b) scale factor of fission rate along the BB' line shown in (a) for three different Mo distributions.

Figure 8. Effect of Mo inhomogeneous distributions on swelling kinetics under fission rate $\dot{f}=1.2 \times 10^{20}$ fission $/\left(\mathrm{m}^{3} \mathrm{~s}\right)$ and nucleation factor $f_{n}=0.03$. 
Table 1. Model parameters used in the simulations

\begin{tabular}{|c|c|c|}
\hline Characteristic length & $l_{0}$ & $50 \mathrm{~nm}$ \\
\hline Grid size & $d x=d y=d z$ & $50 \mathrm{~nm}$ \\
\hline Diffusivity of Xe & $D_{g}=D_{0} \dot{f}$ & $D_{0}=1.0 \times 10^{-40} \mathrm{~m}^{5} /$ fission \\
\hline Grain boundary energy & $\gamma$ & $1.0 \mathrm{~J} / \mathrm{m}^{2}$ \\
\hline Yield of Xe per fission & $\beta$ & 0.25 \\
\hline Fission rate & $\dot{f}$ & $1.2 \times 10^{20} \sim 2.4 \times 10^{22}$ fission $/ \mathrm{m}^{3} / \mathrm{s}$ \\
\hline $\begin{array}{l}\text { Grain boundary } \\
\text { enhance factor }\end{array}$ & $\xi$ & $15 \sim 125$ \\
\hline Dissolution rate & $b=b_{0} \dot{f}$ & $b_{0}=2.0 \times 10^{-24}$ \\
\hline $\begin{array}{l}\text { Ver der Waals constant } \\
\text { for Xe }\end{array}$ & $b_{v}$ & $8.5 \times 10^{-29}$ \\
\hline Nucleation factor & $f_{n}$ & $0.01 \sim 0.03$ \\
\hline $\begin{array}{l}\text { Fitting factor of EOS } \\
\text { for } \mathrm{Xe}\end{array}$ & $h_{s}$ & 0.6 \\
\hline Grain boundary & $\delta$ & $1 \mathrm{~nm}$ \\
\hline
\end{tabular}




\begin{tabular}{|l|l|l|}
\hline thickness & & \\
\hline Grain boundary & $\mu_{G B}$ & $0.0 \mathrm{eV} /$ atom \\
\hline Xe atomtial & $r_{g}$ & $2.16 \times 10^{-10} \mathrm{~m}$ \\
\hline Nearest jump sites & $z$ & 8 \\
\hline Xe formation volume & $\varepsilon_{0}$ & $1.05 \times 10^{29} \mathrm{~m}^{3}$ \\
\hline
\end{tabular}

Table 2 Morphology of four polycrystalline structures

\begin{tabular}{|c|c|c|c|}
\hline & $\begin{array}{c}\text { Number of } \\
\text { grains }\end{array}$ & $\begin{array}{c}\text { Average } \\
\text { Diameter }\end{array}$ & Aspect ratio \\
\hline Crystal A & 548 & $1.34 \mu m$ & $1: 1$ \\
\hline Crystal B & 20 & $4.36 \mu m$ & $1: 1$ \\
\hline Crystal C & 20 & & $4: 1$ \\
\hline Crystal D & 20 & & $16: 1$ \\
\hline
\end{tabular}




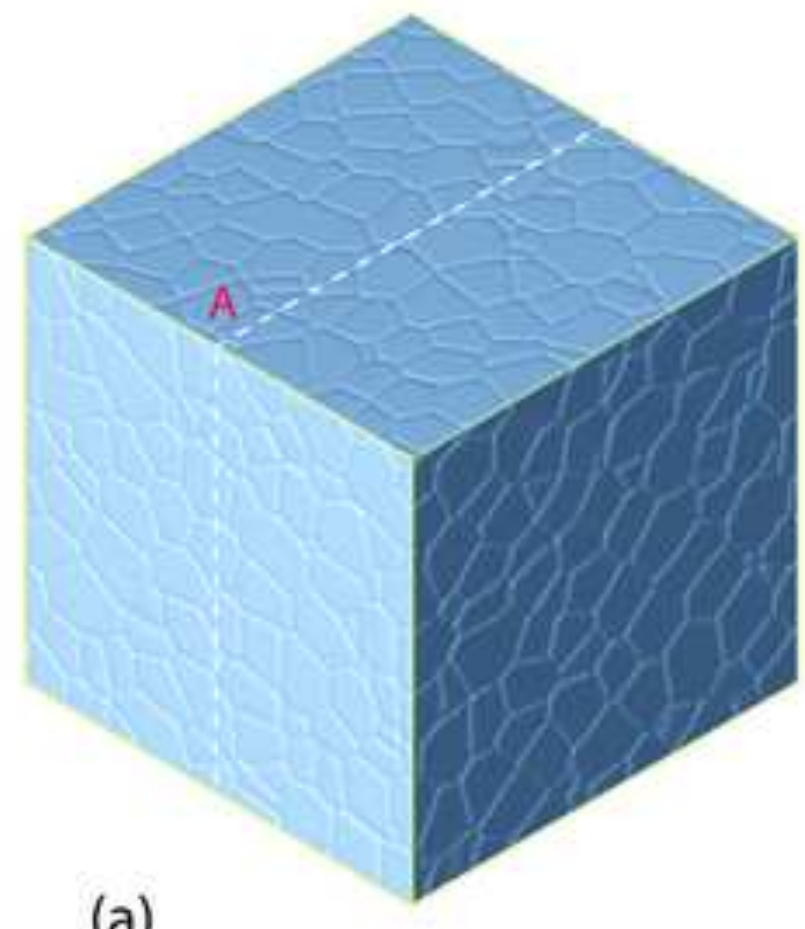

(a)

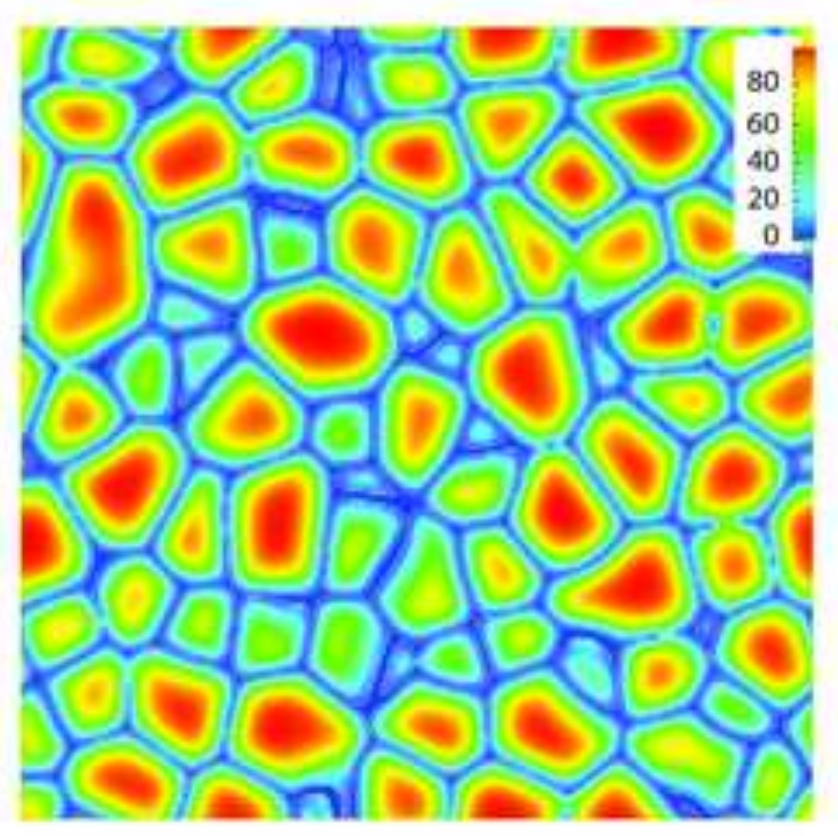

(c)

\section{Figure1}

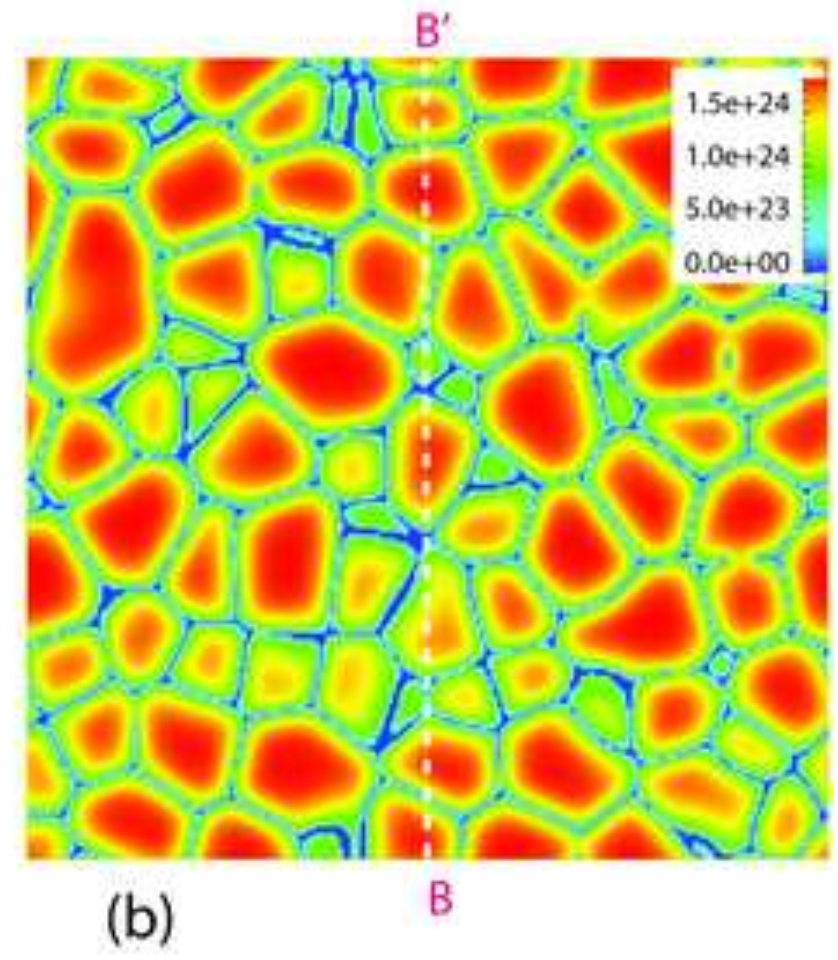

(b)

B

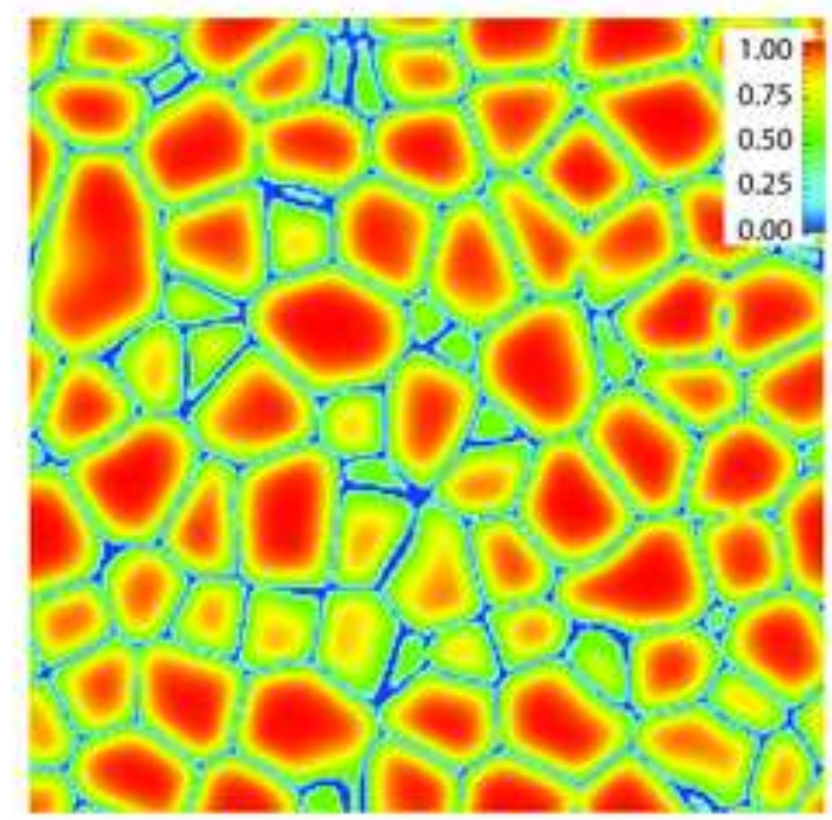

(d)

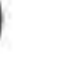




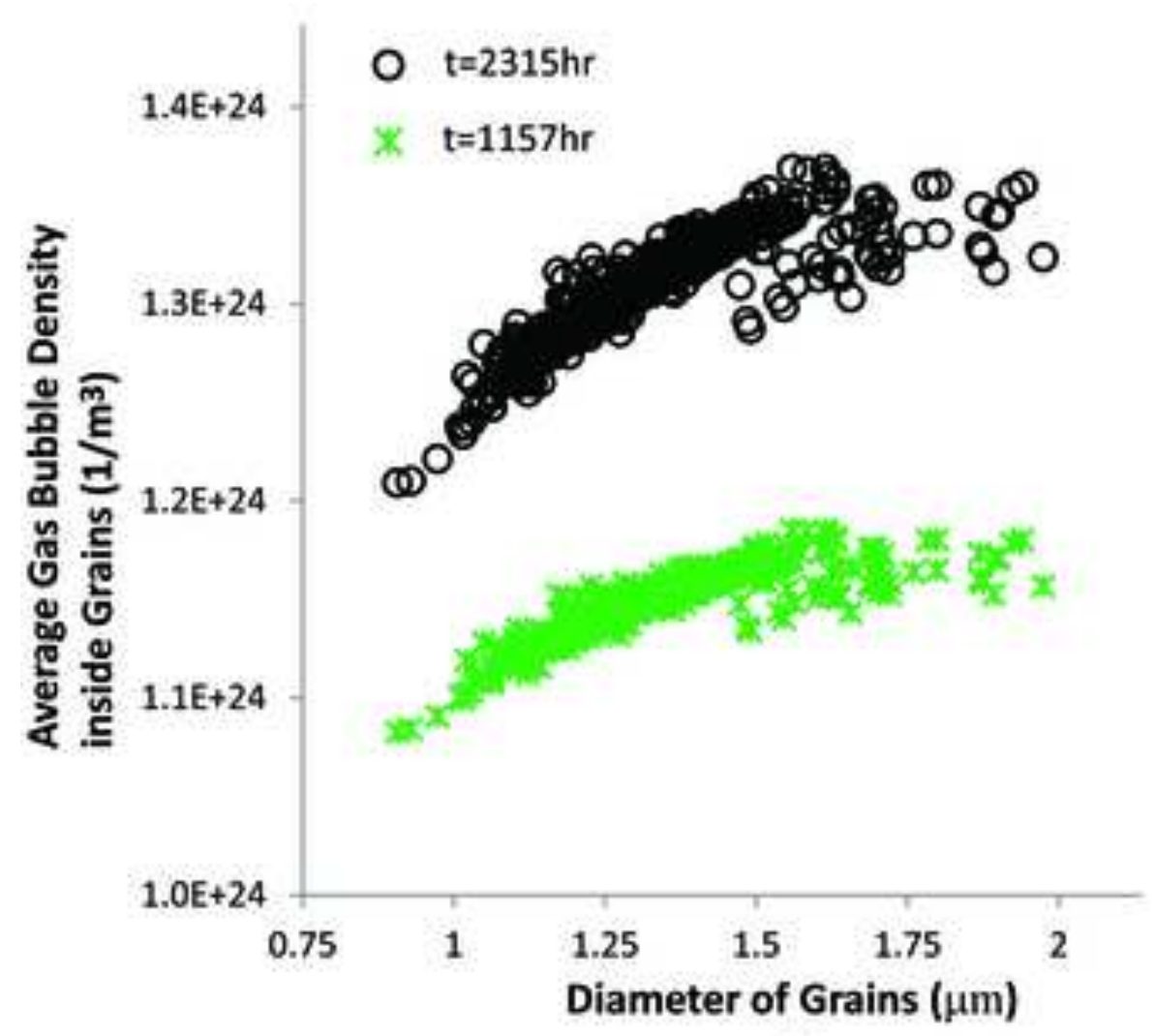

(a)

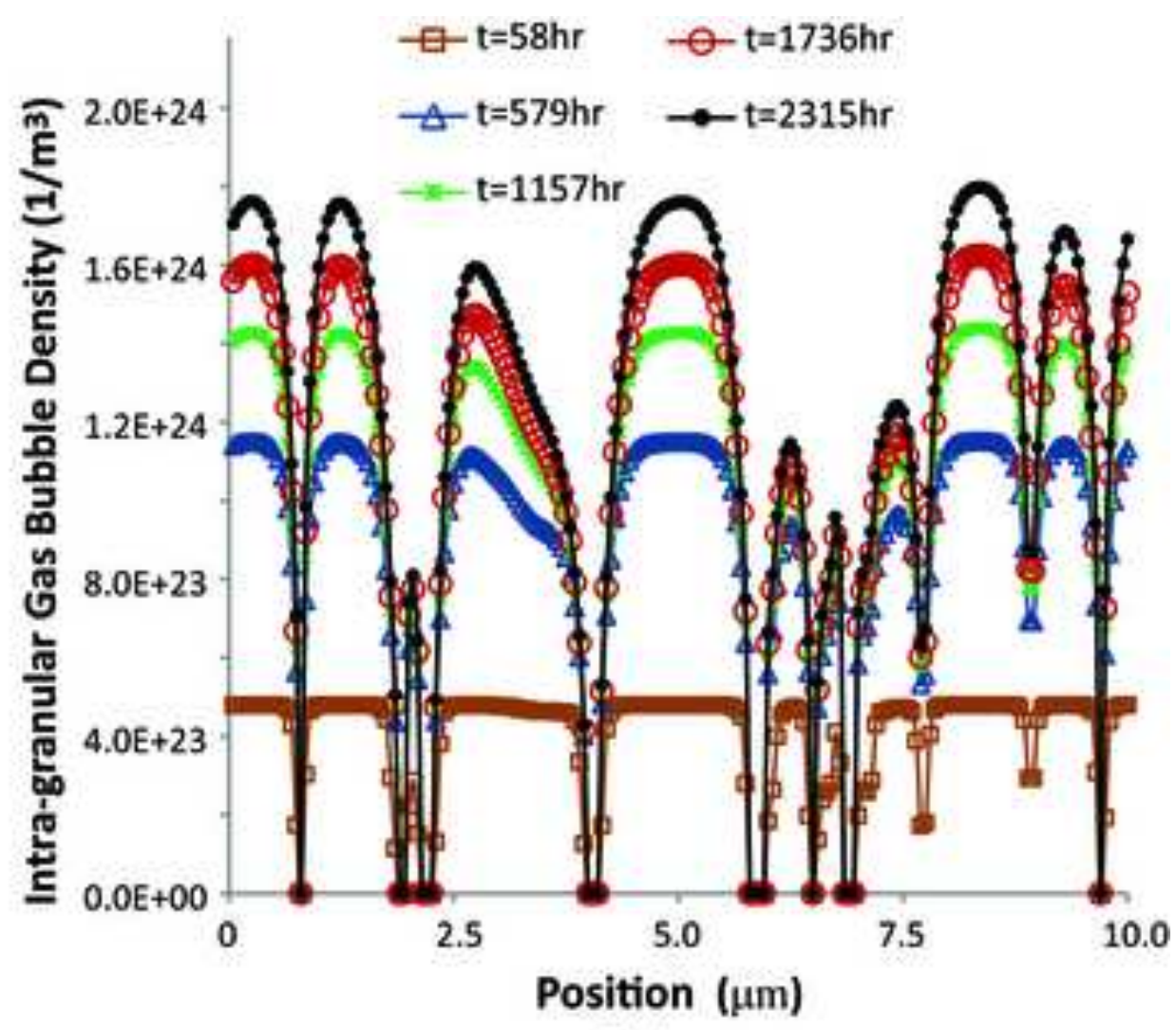

(b) 


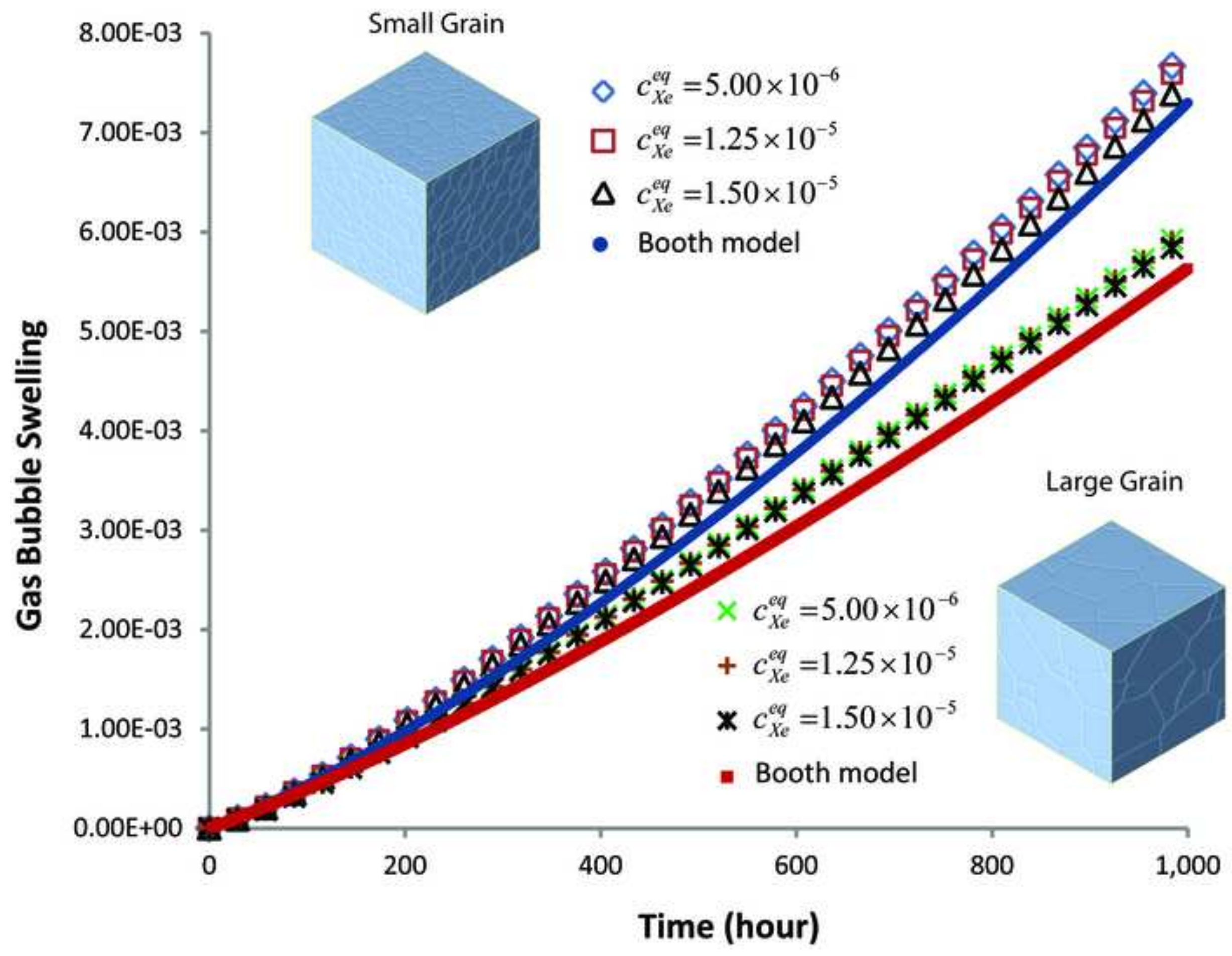




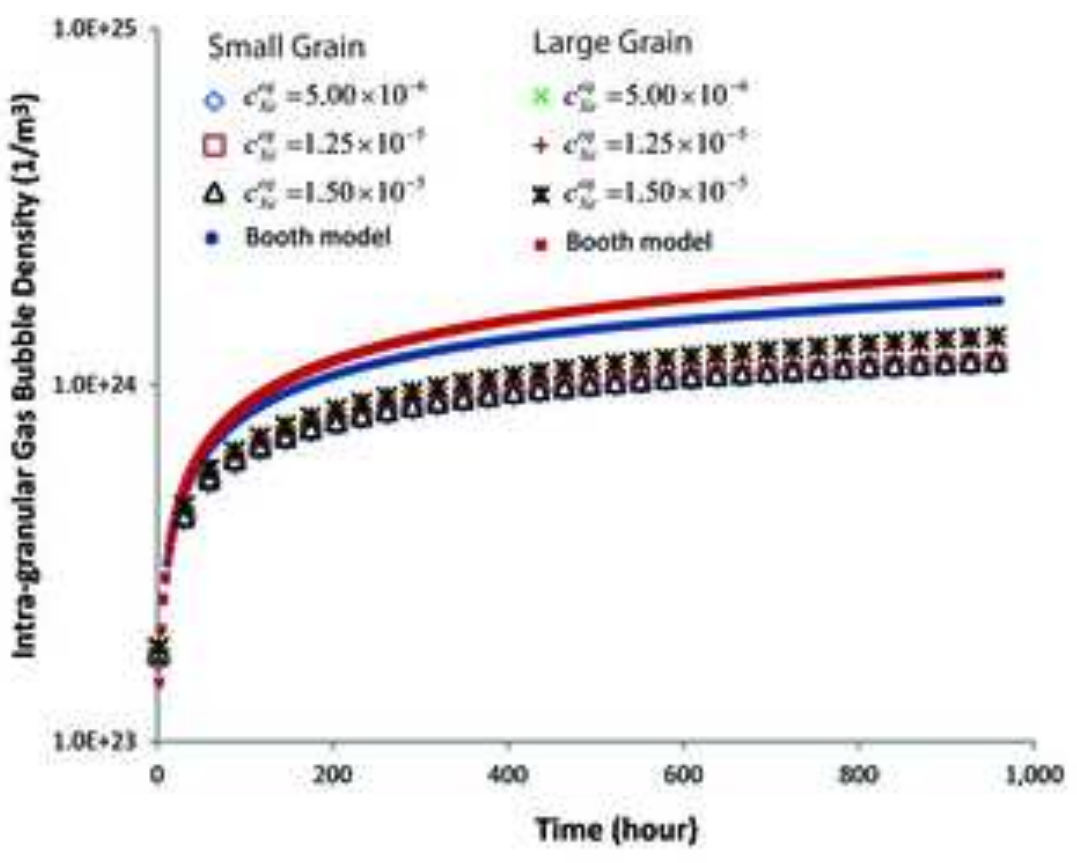

(a)

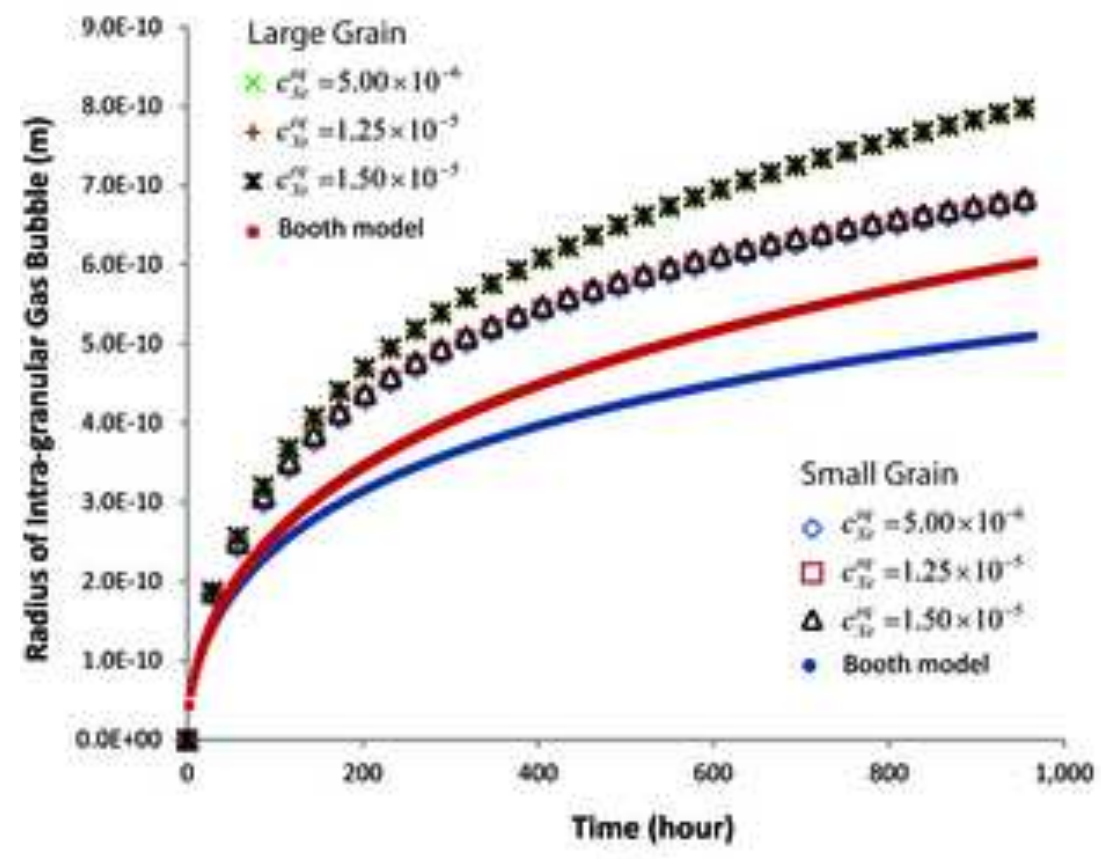

(c)

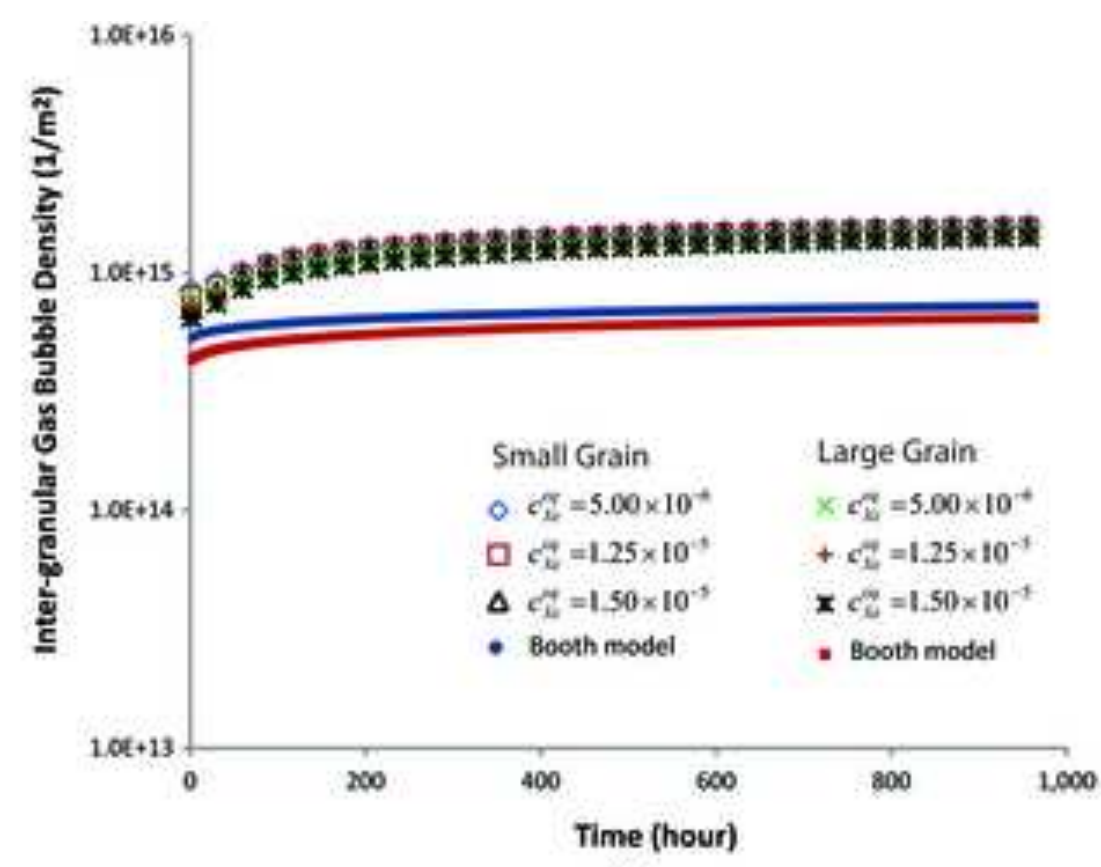

(b)

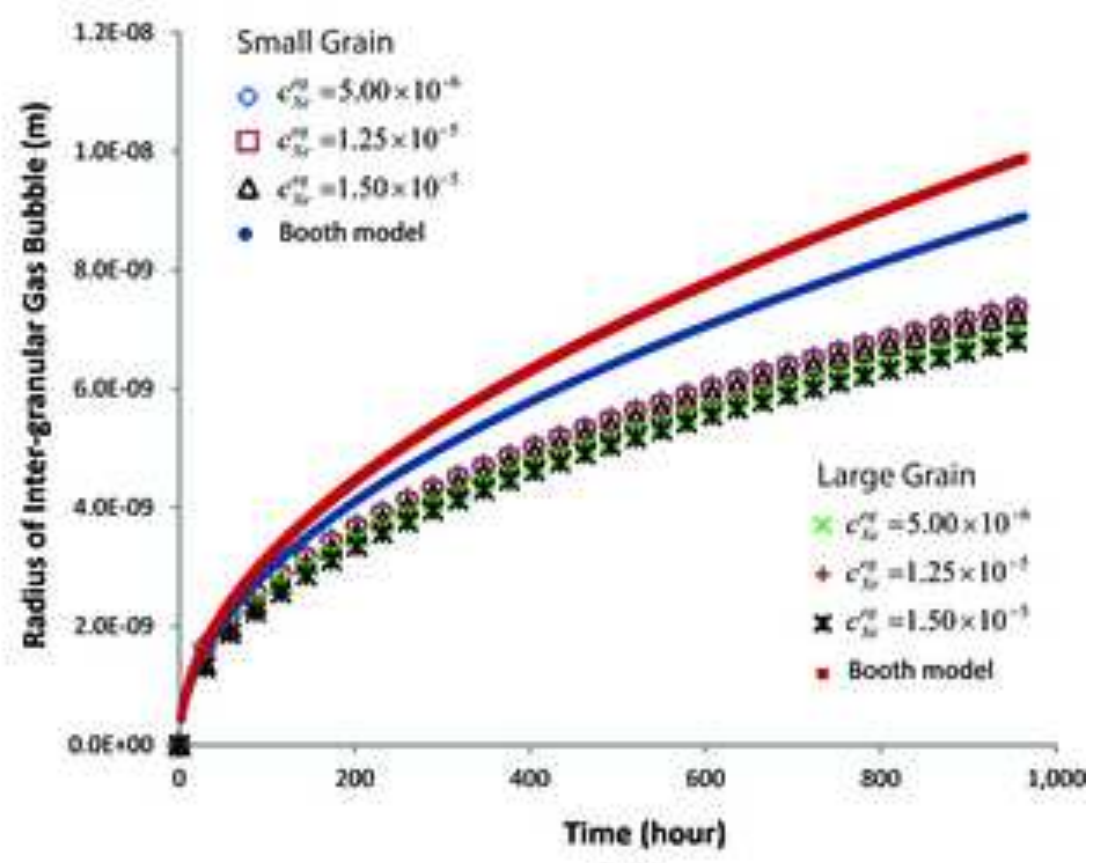

(d) 


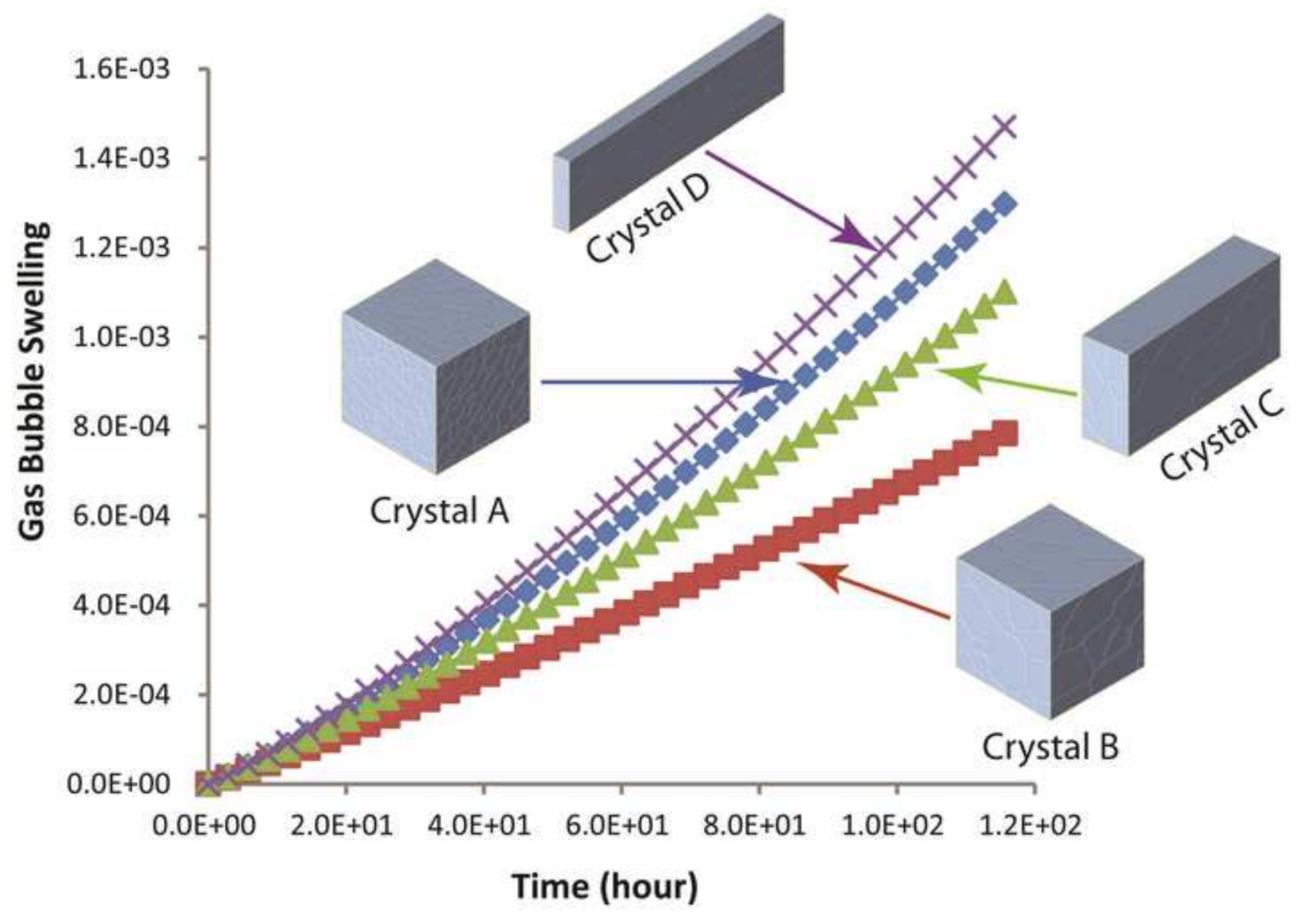




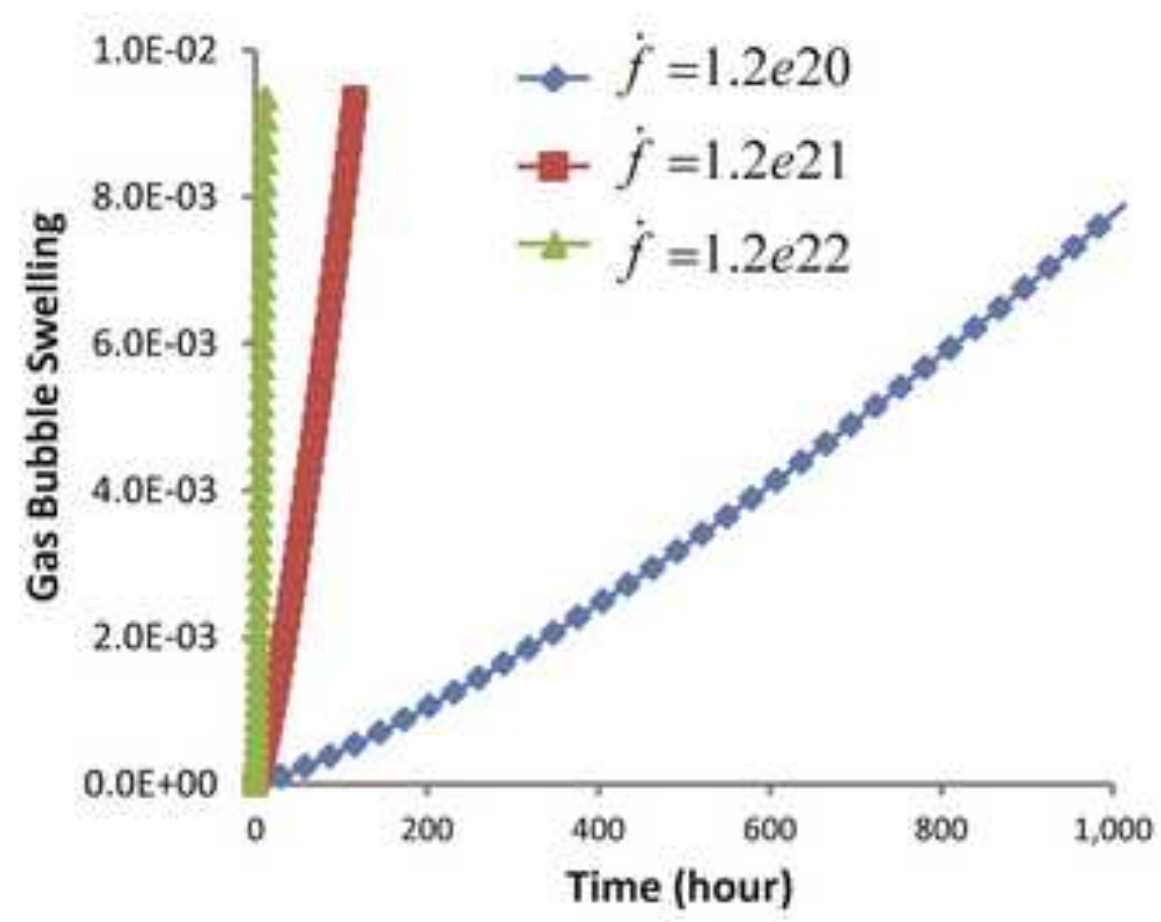

(a)

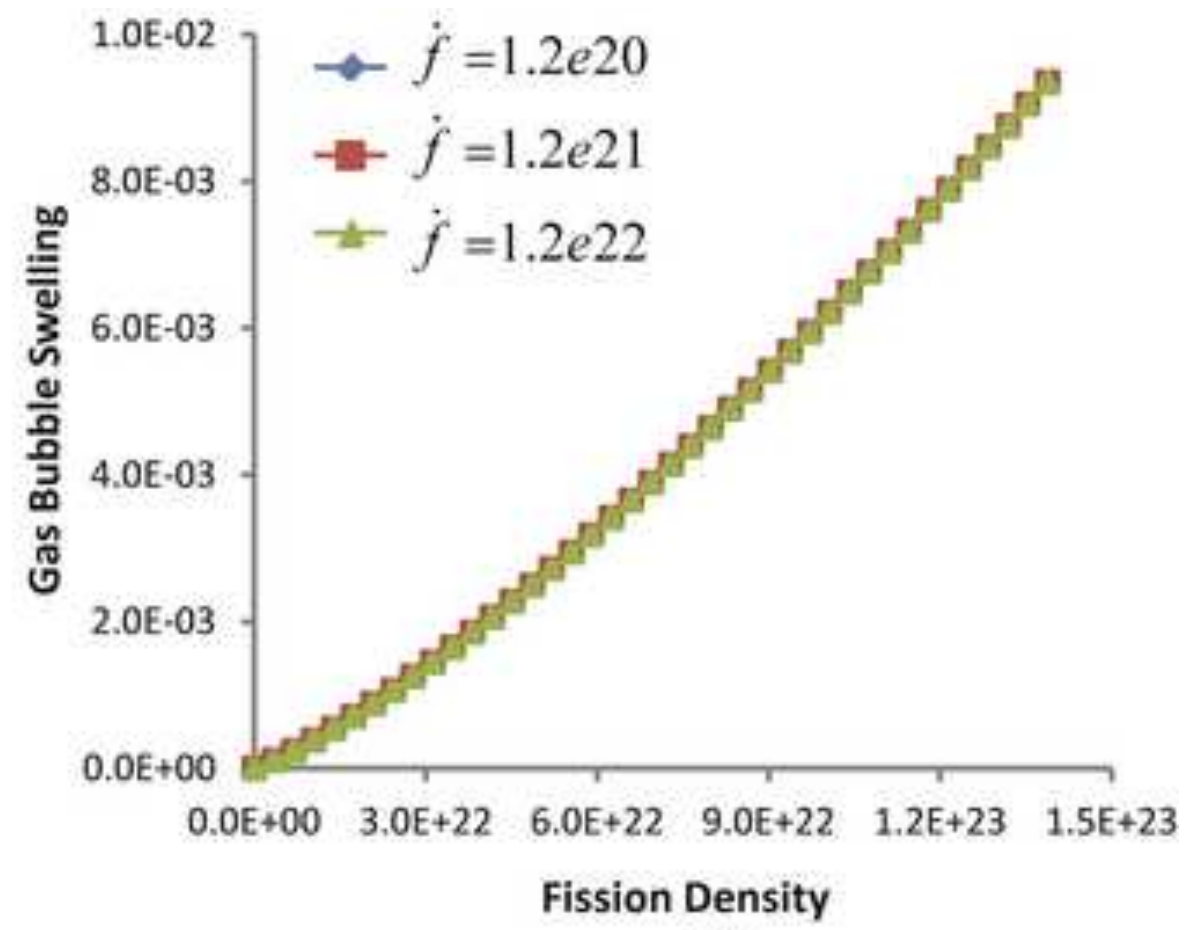

(b) 


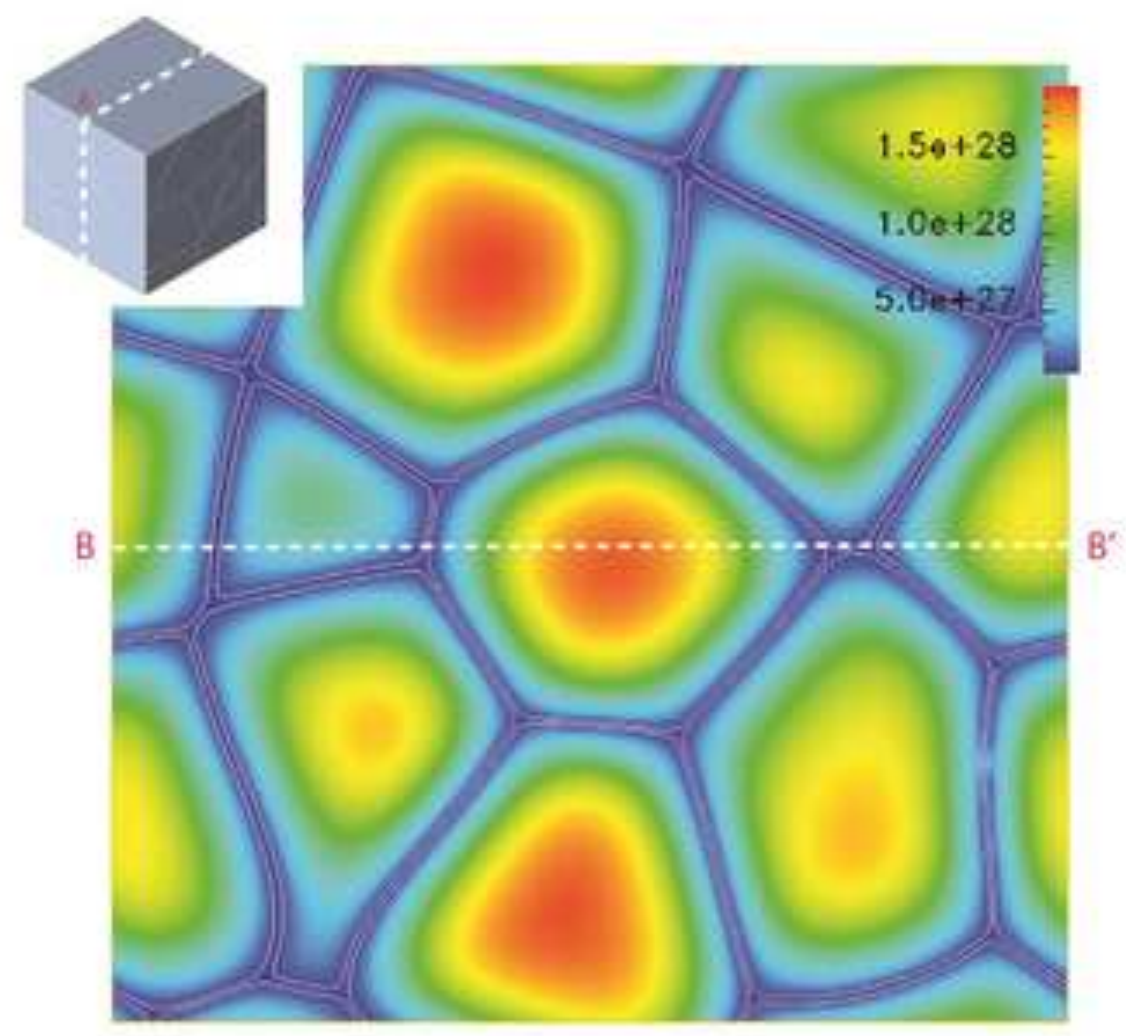

(a)

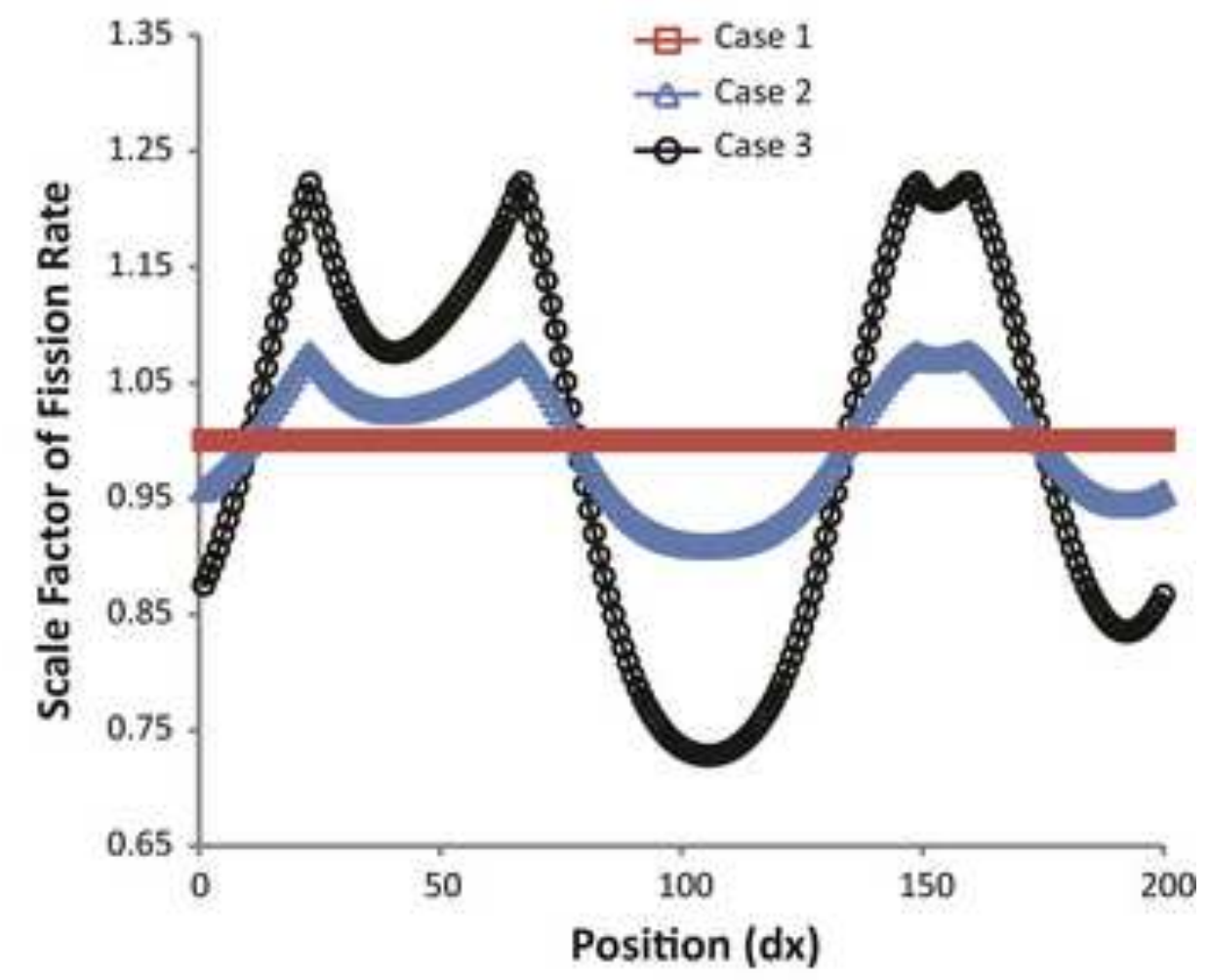

(b) 


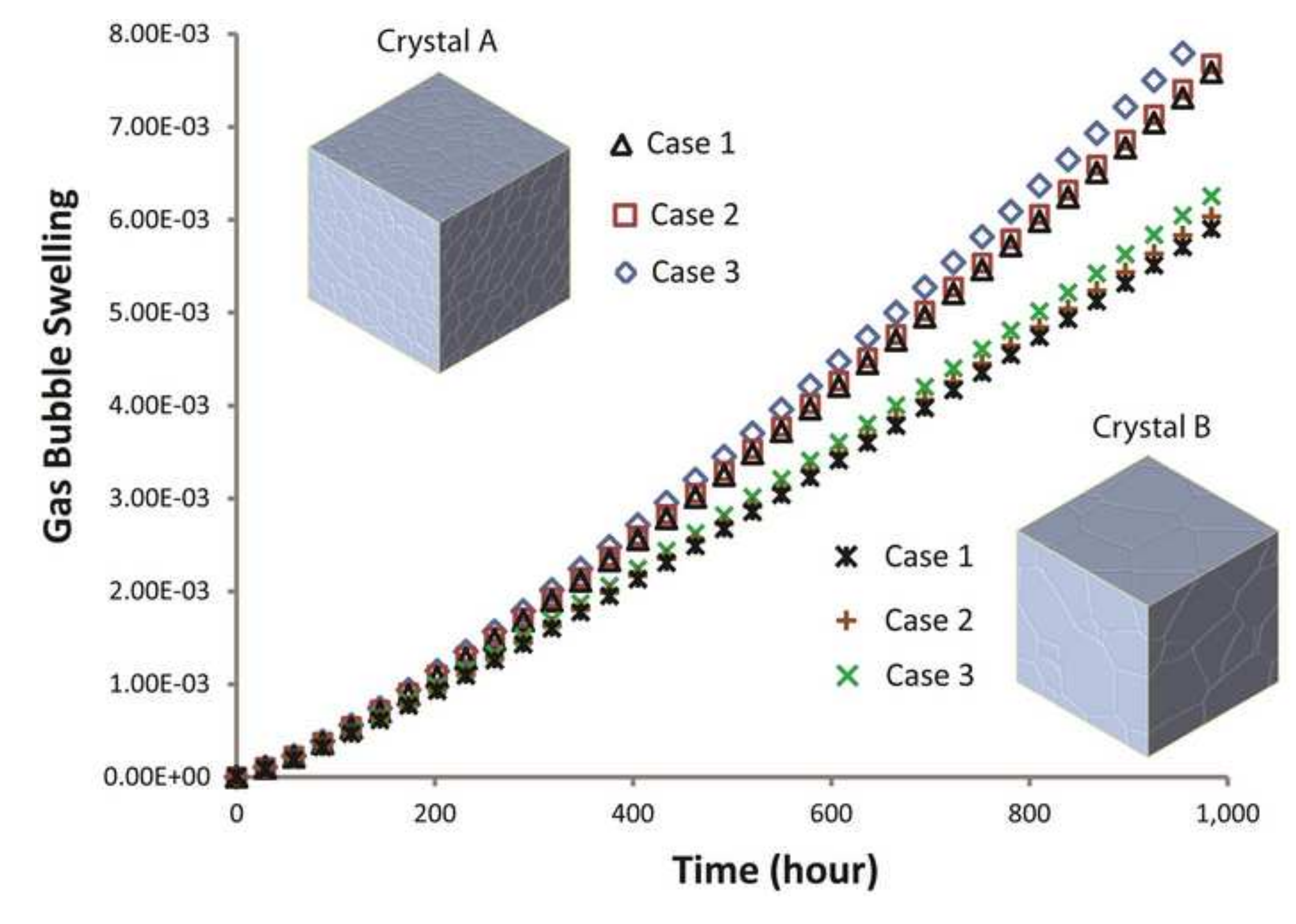

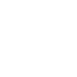

